\title{
Fingerprint image reconstruction for swipe sensor using Predictive Overlap Method
}

\author{
Ahmad Zafrullah Mardiansyah ${ }^{1, *}$, Agus $^{\text {Bejo }}{ }^{1}$, and Risanuri Hidayat ${ }^{1}$ \\ ${ }^{1}$ Department of Electrical Engineering and Information Technology, Universitas Gadjah Mada, Yogyakarta, Indonesia.
}

\begin{abstract}
Swipe sensor is one of many biometric authentication sensor types that widely applied to embedded devices. The sensor produces an overlap on every pixel block of the image, so the picture requires a reconstruction process before heading to the feature extraction process. Conventional reconstruction methods require extensive computation, causing difficult to apply to embedded devices that have limited computing process. In this paper, image reconstruction is proposed using predictive overlap method, which determines the image block shift from the previous set of change data. The experiments were performed using 36 images generated by a swipe sensor with $128 \times 8$ pixels size of the area, where each image has an overlap in each block. The results reveal computation can increase up to $86.44 \%$ compared with conventional methods, with accuracy decreasing to $0.008 \%$ in average.
\end{abstract}

\section{Introduction}

The fingerprint is still a biometric authentication standard that is still growing, especially in the industrial environment [1]. Today's development of technology likely tends to mobile computing. However, many mobile devices on the market have limited ability, especially regarding data processing. In general, fingerprint authentication requires a relatively large computation specification as a minimum. Consequently, fingerprint authentication only applied to devices with standard process capabilities only.

There are many implementations of fingerprint authentication on mobile devices due to the limitations of computing capabilities owned by mobile devices, especially on embedded devices. Efficiency in the computational process of fingerprint authentication system is still developed by many researchers [1-5]. The proposed method and algorithm were helping the development of implementation a fingerprint into embedded devices.

Unlike conventional fingerprint sensors (full sensor) [6], embedded devices have relatively small in sizes [7, 8]. This affect difficulty in implementation process by manufacturers. To overcome these challenges, fingerprint sensor has developed a swipe model that has a smaller size [1]. The swipe type sensor performs a fingerprint scan of every n-pixel (block) of the entire surface in vertically [9]. The results of the reconstruction produced in the research [6] show an overlapping ratio of $84 \%$ match.

Image mosaicking is comparing two images whether it has a correlation or not, and the correlation indicates a shift from one block image to another or vice versa [10]. Although the system successfully deployed, the computational likely leads to be heavy. In the real environment, there will be a delay during the authentication process. There is a time range during finger registration and matching results.

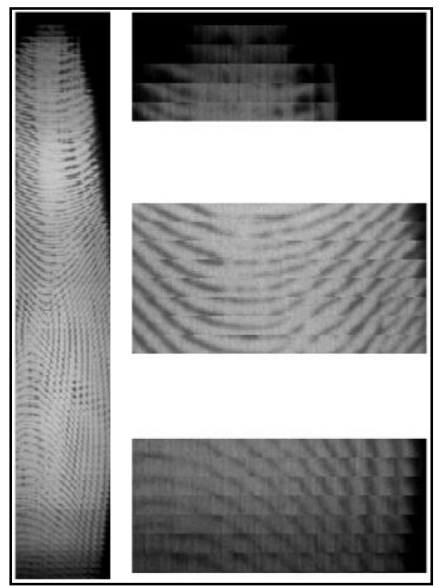

Fig. 1. Raw image from swipe sensor.

Regarding physical dimensions, swipe sensor is more advantageous because it has small in size that is enough to be implemented in embedded devices. However, on the other hand, swipe sensor needs one more process called reconstruction. This process has to reconstruct raw image data from the sensor to become a complete fingerprint image to perform extraction and matching features [11].

The reconstruction process requires a costly computational load. To solve the problem, many researchers have proposed methods and algorithms to reduce computational burdens. The method used in [3] reads pixel of the image by simultaneously to decrease the number of iterations. The relationship between phases of each block was introduced in [12] which was used to

\footnotetext{
* Corresponding author: zaf.mti15@mail.ugm.ac.id
} 
indicate the pixel block correspondence, and the results reveal validated match regardless of computation.

Another research with the same approach was performed by [13] using the Sum of Absolute Difference $(S A D)$ and Fast Normalized Cross Correlation (FastNCC) algorithms. The method shows an accuracy increase of about $1 \%$ using the FastNCC algorithm, but in implementation, FastNCC needs more computational load than SAD because of the number of iterations. In research [14] hybrid image mosaicking has been developed using Mean Absolute Error $(M E A)$ to find the overlap transition value between two images. Mosaicking performed with a combination of pixels and sub-pixels.

From researchers above, there are two critical parameters relating to the performance of fingerprint authentication system. Increased accuracy tends to lead to increased in computational loads and vice versa. The combination of both parameters is capable of producing a better authentication system, but it is still difficult to apply. Nevertheless, research [15] proposed two levels of security with a combination of fingerprint and PIN instruments. Matching on fingerprints is not done based on the features found on the fingerprint, but can be solved using several techniques such as pressure on the sensor surface and the pixel image spreading rate (histogram).

Based on research in computation and accuracy produced by the conventional algorithm, in this paper proposed reconstruction algorithm using predictive overlap method which is used to reduce computation cost. This method applies shift value of previously calculated results to use as current shift value between two blocks; therefore the program does not need to calculate all change values from all blocks by visiting all pixel data.

The paper will be divided into several sections as follows: Section II describes the comparison of conventional reconstruction techniques with the proposed predictive overlap method. The result of experiments and discussions outlined in section IV. The conclusions described in section $\mathrm{V}$.

\section{Reconstruction Algorithm}

\subsection{Conventional Method}

The digital image is composed of a collection of pixels in the form of a two-dimensional form, which is $x$ and $y$. A block of the pixel is considered to be overlapping when its neighboring block has a pixel intensity composition that is mostly the same. Differences in pixel intensity in the block occurs due to differences in the speed of fingerprint shift when the process of acquiring images.

Reconstruct the swipe fingerprint image is to change the position of each block according to their real shift. A value of block's change can be resolved by comparing current block with the previous one; this can be done by calculating the difference between the total intensity of pixels in a block using $S A D$.

$S A D$ needs to visit every pixel in the block while performing calculations, resulting in a computational load of $O(n)$. Matching is done by adjusting the magnitude of the shift $\left(d_{y}\right)$ between two blocks to the maximum block height. The change with the lowest $S A D$ value is the best overlap shift. Next, perform a reconstruction based on the generated in each iteration of the pixel block in the image.

\subsection{Proposed Method}

In practice, the $d_{y}$ value tends to change insignificantly from the previous $d_{y}$ value. The research in this paper utilizes the $d_{y}$ data set of past calculations to determine the probability of the next $d_{y}$ value.
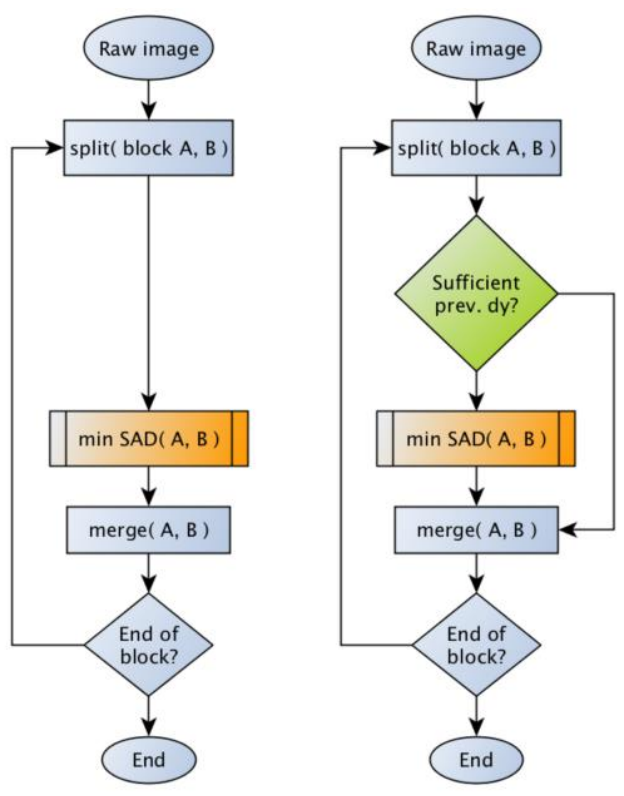

Fig. 2. Conventional (left) and proposed (right) method flow.

From the flowchart in figure 2 above, min SDA process using a large computing cost, the iteration process is done at the pixel level.

$$
S A D=\sum_{i=1}^{n}\left|A_{i}-B_{i}\right|
$$

where $A$ and $B$ are the two block that will be compared by finding the difference between pixel level, is the accumulation of the difference between block $A$ and $B$.

The proposed approach adds a checking process to the previous $d_{y}$ values. In this paper, the reference value is two sets of former $d_{y}$ (range), then the value will be used as $d_{y}$ in the next predictive overlap $\left(d_{y \text {-pred }}\right)$ by making it as a variable (count). The value of $d_{y \text {-pred }}$ obtained by calculating the middle value between the two former $d_{y}$. By minimizing min $S D A$ process, it indirectly reduces the computational load required in reconstruction overall process.

$$
d_{y-\text { pred }}=\frac{d_{y i-1}+d_{y i-2}}{2}
$$

Where $d_{y i-1}$ is the first previous $d_{y}$ value, and $d_{y i-2}$ is the second last $d_{y}$ value, $d_{y-\text { pred }}$ is the predicted value obtained by calculating the average of both former $d_{y}$. 


\section{Results and Discussion}

The experiments are done by reconstructing the set of fingerprint images captured using sensor swipe. Reconstruction is divided into two parts, first for the original and second after applying the predictive overlap method and adjusting the count variables.

There are 36 raw image data used in this experiments, images obtained from swipe sensor with six different fingers and six different shooting for each corresponding finger. In the original version, raw images reconstructed without using predictive overlap method.
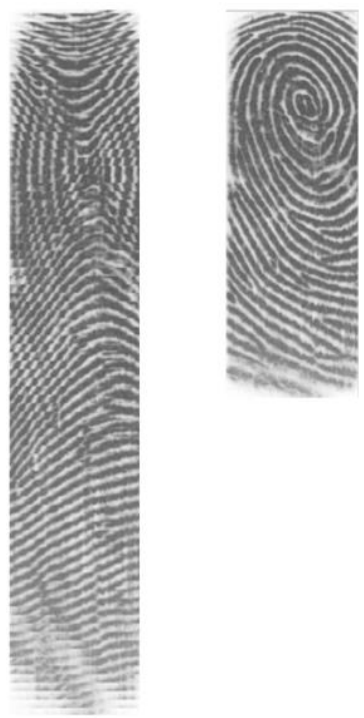

Fig. 3. Raw image from swipe sensor (left), original reconstructed image (right).

Reconstruction performed on all raw image data using predictive overlap method. Figure 4 shows the percentage of comparison between the computational time required by conventional reconstruction algorithm with reconstruction using predictive overlap approach. In the rebuilding process, the count variable indicates how often $d_{y-\text { pred }}$ used.

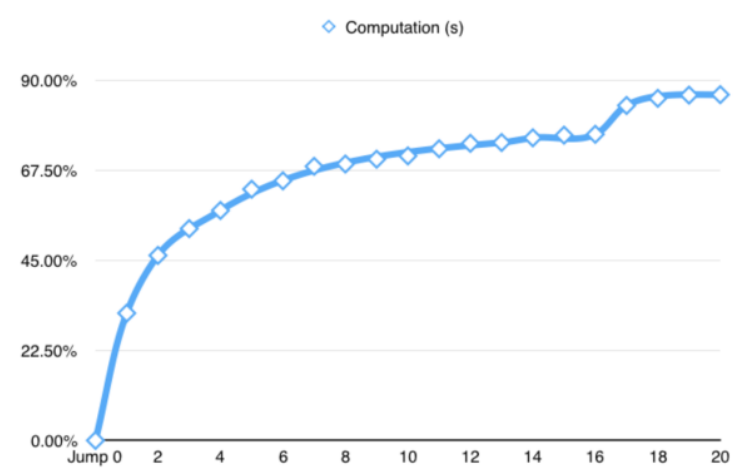

Fig. 4. Comparison computational time between conventional and predictive overlap.

By applying predictive overlap, the computational load tends to decrease in every turn of the count variables. When the count value equals 20 , this method can reach $86.44 \%$ increase in computation time.

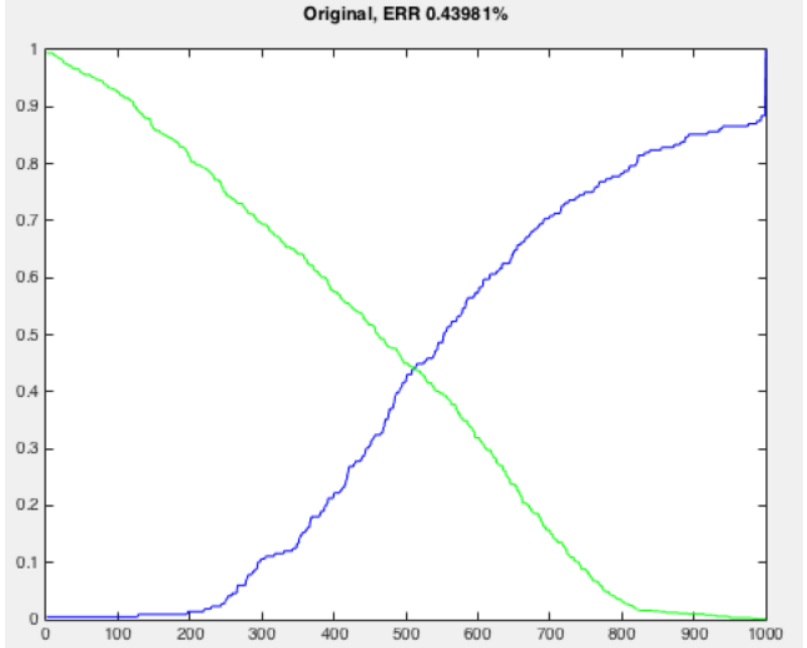

Fig. 5. EER for original reconstruction.

Next experiment is measuring the accuracy of the reconstructed image by matching the same finger (genuine) and the different finger (impostor). In figure 5, the original reconstructed image yields accuracy with EER $0.4398 \%$.

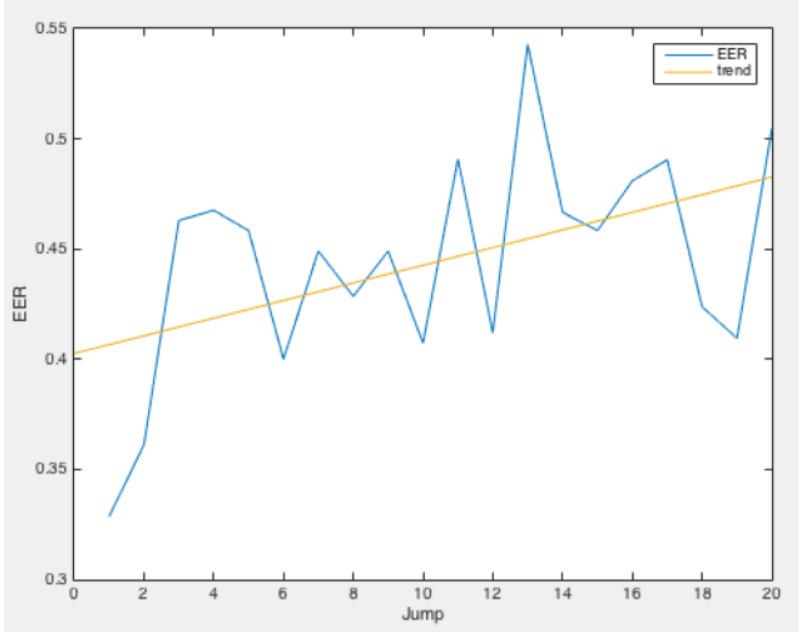

Fig. 6. Accuracy level by applying predictive overlap method.

On the other hand, with increasing in computational time, the accuracy of reconstructed images tends to decrease. As seen in figure 6 above, EER is fluctuating but shows an increasing tendency. The average $E E R$ obtained from all count values is $0.4446 \%$, in other words, the $E E R$ rate increases by $0.0048 \%$.

Variations in accuracy were caused by the difference of the extracted minutiae (feature) from the pattern on the reconstructed image. Figure 7 (left) is the result of conventional algorithm reconstruction, the number of minutiae features found is 27 . Figure 7 (center) and (right) are the result of reconstruction with predictive overlap method, the number of minutiae features extracted respectively are 24 and 26 . The reason is an incompatibility in placing block pixels while performing reconstruction, which is used to estimate the possibility of the next shift value. 

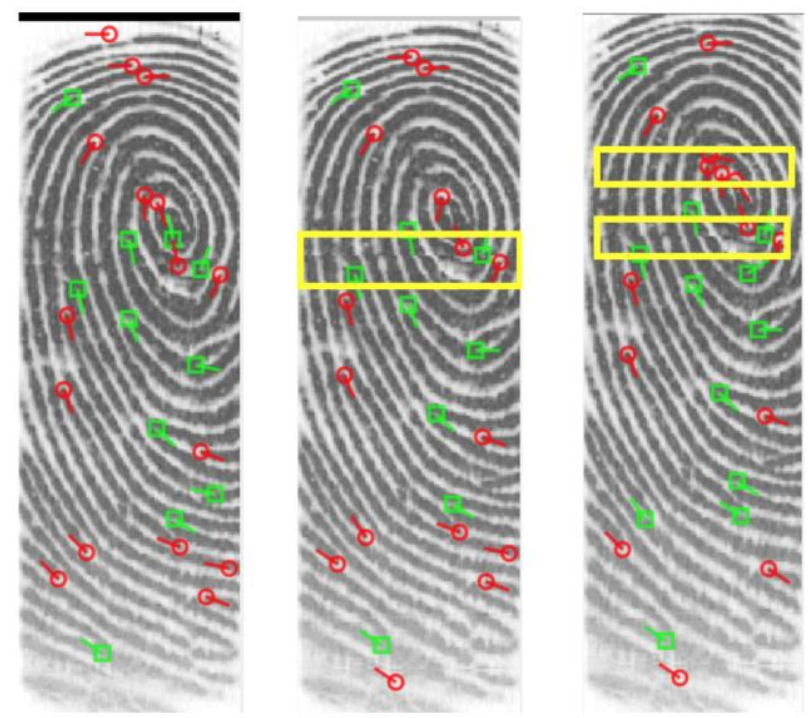

Fig. 7. Extracted minutiae for original image (left), predictive overlap with count $=10$ (center), count $=20$ (right).

Conducted experiments reveal that increasing in computation time, even by using the same $d_{y}$ for 20 times, while the changes in accuracy during the count tend to be stable. Thus this method is suitable to apply to devices that have limitations in the computing process.

\section{Conclusion}

In this paper, experiments of the predictive overlap method have successfully reduced the computational load for swipe fingerprint images. Increased computing time has no significant impact on matching accuracy. The iterations performed in the predictive overlap method based on the previous set of $d_{y}$, called $d_{y \text {-pred }}$. From the test results, obtained computation time can increase up to $86.44 \%$, with decreasing in accuracy only $0.0048 \%$.

\section{References}

1. Kanich, O. and M. Drahanský, Currently Used Swipe Fingerprint Sensors. International Journal of BioScience and Bio-Technology, 2016. 8(1): p. 381-386.

2. DABBAH, M.A., W.L. WOO, and S.S. DLAY, Computationally Efficient Fingerprint Algorithm for Automatic Recognition. 2005. ilseps

3. Hamine, M., Y. Audet, and J.-P. David, a Real Time Image Reconstruction Algorithm For An Integrated Fingerprint Sensor. 2007.

4. Li, G., C. Busch, and B. Yang, A novel approach used for measuring fingerprint orientation of arch

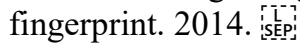

5. Kasban, H., Fingerprints verification based on their spectrum. Neurocomputing, 2016. 171: p. 910-920.

6. Lee, J.-W., et al., A 600-dpi Capacitive Fingerprint Sensor Chip and Image-Synthesis Technique. 1999.

7. Xia, X. and L. O'Gorman, Innovations in ngerprint capture devices. 2003. iș
8. Kamaraju, M. and P.A. Kumar, DSP based Embedded Fingerprint Recognition System. 2013.

9. Clausen, S., A Single-Line AC Capacitive Fingerprint Swipe Sensor. [íme

10. Habegger, A., et al., A Subpixel-Based Fingerprint Reconstruction Algorithm. 2012. 'í

11. Galy, N., B.T. Charlot, and B. Courtois, A Full Fingerprint Verification System for a Single-Line

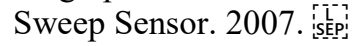

12. Jie, L., H. Xuxiao, and W. Puyu, Sequence Fingerprint Image Mosaic Method and Its Experimental Study Based on Phase Correlation and

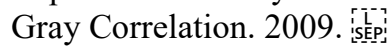

13. TAN, S.-Y., et al., A Sweeping Fingerprint Verification System using the Template Matching

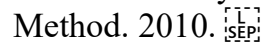

14. Zhang, Y.-1., J. Yang, and H.-t. Wu, a Hybrid Swipe Fingerprint Mosaicing Scheme. 2005. is sép.

15. Jacomet, M., J. Goette, and A. Eicher, On Using Fingerprint-Sensors for PIN-Pad Entry. 2008. 